\title{
Concentração Analgésica Mínima da Bupivacaína Durante Infusão Peridural Contínua após Bloqueio Subaracnóideo no Período Pós-Operatório de Cirurgias Ortopédicas da Perna, Tornozelo e Pé *
}

\section{Minimum Analgesic Concentration of Bupivacaine After Continuous Epidural Infusion Following Spinal Anesthesia in the Postoperative Period of Leg, Ankle and Foot Surgery}

Getúlio Rodrigues de Oliveira Filho, TSA ${ }^{1}$, Nilton Gesser, TSA ${ }^{2}$, Márcia Regina Ghellar, TSA ${ }^{3}$, Ranulfo Goldschmidt, TSA ${ }^{2}$, Adilson José Dal Mago, TSA ${ }^{2}$

\section{RESUMO}

Oliveira Filho GR, Gesser N, Ghellar MR, Goldschmidt R, Dal Mago AJ - Concentração Analgésica Mínima da Bupivacaína Durante Infusão Peridural Contínua após Bloqueio Subaracnóideo no Período Pós-Operatório de Cirurgias Ortopédicas da Perna, Tornozelo e Pé

Justificativa e Objetivos - A concentração analgésica mínima de um anestésico local (CAM-AL) corresponde à concentração efetiva em $50 \%$ das pacientes durante o primeiro estágio do trabalho de parto. Pode ser utilizada para determinar a potência relativa de diferentes agentes e estimar o efeito de drogas analgésicas co-administradas no espaço peridural. O objetivo deste estudo foi o de determinar a CAM-AL da bupivacaína para analgesia peridural de cirurgias ortopédicas.

Método - Foi aplicada a técnica de alocação seqüencial não aleatória duplamente encoberta a 23 adultos submetidos a cirurgias ortopédicas sobre a perna, tornozelo ou pé. A anestesia constou de bloqueio subaracnóideo lombar com bupivacaína hiperbárica. Um cateter peridural colocado em $L_{4}-L_{5}$ foi avançado 3 a $5 \mathrm{~cm}$ em direção cefálica. No período pós-operatório imediato, foram administrados $20 \mathrm{ml}$ de bupivacaína seguida de infusão de $0.15 \mathrm{ml} \cdot \mathrm{kg}^{-1} \cdot \mathrm{h}^{-1}$, na concentração apropriada. Escores analógicos visuais de dor e de Bromage foram registrados após 4, 8 e 12 horas. A concentração foi considerada eficaz quando os escores de dor foram inferiores a $10 \mathrm{~mm}$ em todas as avaliações. A concentração inicial foi de $0,3 \%$ e diminuiu ou aumentou $0,1 \%$ caso a resposta do paciente anterior tenha sido ineficaz ou eficaz, respectivamente. A CAM-AL foi calculada pela fórmula de Massey e Dixon.

Resultados - A CAM-AL da bupivacaína (limites de 95\% de confiança) foi de $0,16 \%(0,11 \%$ e $0,21 \%)$. Bloqueio motor intenso foi observado na maioria dos pacientes.

Conclusões - Para uma taxa de infusão de $0.15 \mathrm{ml} . \mathrm{kg}^{-1} \cdot \mathrm{h}^{-1}$, a CAM-AL da bupivacaína foi de $0,16 \%$. No entanto, o modelo

* Recebido do (Received from) Hospital Governador Celso Ramos, CET/SBA Integrado de Anestesiologia da SES-SC, Florianópolis, SC

1. Responsável pelo CET/SBA

2. Instrutor do CET/SBA

Apresentado (Submitted) em 03 de janeiro de 2001

Aceito (Accepted) para publicação em 27 de abril de 2001

Correspondência para (Mail to):

Dr. Getúlio Rodrigues de Oliveira Filho

Rua Luiz Delfino 111/902

88.015-360 - Florianópolis, SC

E-mail: grof@th.com.br utilizado pode não ter sido adequado para a avaliação dos efeitos motores das concentrações testadas.

UNITERMOS: ANALGESIA: pós-operatória;ANESTÉSICOS, Local: bupivacaína; TÉCNICAS ANESTÉSICAS, Regional: peridural contínua, subaracnóidea

\section{SUMMARY}

Oliveira Filho GR, Gesser N, Ghellar MR, Goldschmidt R, Dal Mago AJ - Minimum Analgesic Concentration of Bupivacaine After Continuous Epidural Infusion Following Spinal Anesthesia in the Postoperative Period of Leg, Ankle and Foot Surgery

Background and Objectives - Minimum analgesic concentration of a local anesthetic (MAC-LA) is the effective concentration for $50 \%$ of patients $\left(E C_{50}\right)$ during the first stage of labor. It may be used to determine relative analgesic potency and to estimate the effects of co-administered epidural analgesics. This study aimed at determining epidural bupivacaine's MAC-LA for orthopedic surgery.

Methods - A double-blind non randomized sequential allocation method for MAC calculation was applied to 23 adult patients undergoing orthopedic leg, ankle or foot surgeries. Anesthesia was obtained with lumbar spinal hyperbaric bupivacaine. An epidural catheter placed at $L_{4}-L_{5}$ level was inserted 3 to $5 \mathrm{~cm}$ in the cephalad direction. Postoperatively, a 20 $\mathrm{ml}$ epidural bupivacaine bolus followed by $0.15 \mathrm{ml} . \mathrm{kg}^{-1} \cdot \mathrm{h}^{-1}$ infusion were administered at the appropriate concentration. Pain and Bromage scores were recorded after 4, 8 and 12 hours. Bupivacaine concentration was considered effective when visual analog pain scores were below $10 \mathrm{~mm}$ in all evaluations. Initial concentration was $0.3 \%$ and was subsequently decreased or increased by $0.1 \%$ for next patient when previous response was effective or ineffective, respectively. MAC-LA was calculated by Dixon and Massey's formula.

Results - Bupivacaine's MAC-LA (95\% confidence limits) was $0.16 \%(0.11 \%$ and $0.21 \%)$. Intense motor blockade was observed in most patients.

Conclusions - For a $0.15 \mathrm{ml} . \mathrm{kg}^{-1} \cdot \mathrm{h}^{-1}$ infusion rate, bupivacaine's MAC-LA was $0.16 \%$. However, the model may have not been suitable for the evaluation of motor effects of tested concentrations.

KEY WORDS: ANALGESIA: postoperative; ANESTHETICS, Local: bupivacaine; ANESTHETIC TECHNIQUES, Regional: continuous epidural, spinal block

(C) Sociedade Brasileira de Anestesiologia, 2001 


\section{INTRODUÇÃO}

A concentração analgésica mínima de anestésicos locais (CAM-AL) é a concentração eficaz mediana de um anestésico local durante analgesia para o primeiro estágio do trabalho de parto. Portanto, corresponde à concentração de anestésico local administrado no espaço peridural que produz analgesia em $50 \%$ das pacientes $\left(\mathrm{CE}_{50}\right)^{1}$. O modelo para cálculo da CAM-AL em obstetrícia tem permitido a determinação das potências relativas de diferentes anestésicos locais ${ }^{1-3}$ e o efeito da administração concomitante de opióides por via peridural ${ }^{4-7}$.

A CAM-AL é calculada pelo método de alocação seqüencial não aleatória descrito por Massey e Dixon, sendo a concentração do anestésico local administrada a cada paciente, condicionada à resposta do paciente anterior ${ }^{1,8,9-11}$. Entre as vantagens deste método, estão a diminuição entre 30 e $70 \%$ do tamanho da amostra em comparação aos estudos com múltiplos grupos com concentrações fixas do anestésico local, além da redução do número de pacientes expostos a analgesia inadequada ${ }^{1,8}$.

As cirurgias ortopédicas sobre os membros inferiores provocam dor pós-operatória de intensidade moderada a forte, o que justifica a infusão peridural contínua ou intermitente de anestésicos locais associados ou não a opióides, no período pós-operatório imediato. Entretanto, ainda não foi definida a concentração analgésica mínima dos anestésicos locais para este tipo de cirurgia. O conhecimento da CAM-AL destes anestésicos em cirurgias ortopédicas do membro inferior pode ser útil para comparar a potência relativas dos anestésicos e para estudar o efeito de drogas adjuvantes.

Assim, este estudo teve por objetivo determinar a CAM-AL da bupivacaína para infusão peridural contínua no período pós-operatório de cirurgias ortopédicas dos membros inferiores.

Para definir o tamanho da amostra, os escores analógicos visuais de dor após cirurgias ortopédicas do membro inferior e o intervalo entre as concentrações de bupivacaína a ser utilizado para determinação da CAM-AL (Estudo II), foi realizado um estudo piloto, o Estudo I.

\section{MÉTODO}

Ambos os estudos tiveram a aprovação da Comissão de Ética Médica do Hospital Governador Celso Ramos e foi obtido o consentimento após esclarecimento dos pacientes.

Todos os pacientes foram classificados como estado físico ASAI. Foram critérios de exclusão idade inferior a 18 e superior a 65 anos, pacientes não cooperativos, portadores de doenças neurológicas ou psiquiátricas, adição a drogas ilícitas, portadores de distúrbios hepáticos e renais, diabete melito e imobilização gessada da extremidade operada.

Os estudos foram realizados de forma prospectiva e duplamente encoberta.

Os pacientes receberam, como medicação pré-anestésica, diazepam $\left(0,1\right.$ a $\left.0,15 \mathrm{mg} \cdot \mathrm{kg}^{-1}\right)$, por via oral, 60 a 90 minutos antes do horário previsto da cirurgia. Foi instalada venóclise periférica e administração de solução fisiológica à velocidade de $2 \mathrm{ml} \cdot \mathrm{kg}^{-1} \cdot \mathrm{h}^{-1}$. A monitorização incluiu cardioscópio, monitor não invasivo de pressão arterial e oxímetro de pulso.

\section{Estudo I}

Foram estudados, 28 pacientes, com idade entre 19 e 56 anos, de ambos os sexos, submetidos a cirurgias ortopédicas sobre o membro inferior (quadril e fêmur: 4 pacientes; joeIho: 11 pacientes; perna, tornozelo e pé: 13 pacientes). Foi instalado um cateter peridural em $L_{4}-L_{5}$ e avançado em direção cefálica 3 a $5 \mathrm{~cm}$. Os pacientes foram anestesiados com propofol ( 2 a $2,5 \mathrm{mg} \cdot \mathrm{kg}^{-1}$ ) e óxido nitroso a $50 \%$ e isoflurano, sob ventilação controlada manual através de máscara laríngea. Quando os pacientes recuperaram a consciência, foi registrado o escore analógico visual em uma escala de $100 \mathrm{~mm}$ ( 0 = nenhuma dor, 100 = pior dor imaginável), conforme instrução e treinamento prévios. Caso o escore analógico visual fosse superior a $10 \mathrm{~mm}$, o paciente receberia $20 \mathrm{ml}$ de bupivacaína na concentração adequada, através do cateter peridural. O primeiro paciente recebeu bupivacaína a $0,125 \%$ e os seguintes, receberam concentrações $0,0125 \%$ maiores ou menores, de acordo com a resposta do paciente anterior, conforme descrito a seguir.

A eficácia da concentração analgésica da bupivacaína foi avaliada por um observador que desconhecia a concentração de bupivacaína, 30 minutos após a administração e classificada como:

a) Eficaz: quando o escore analógico visual de dor foi menor ou igual a $10 \mathrm{~mm}$. Neste caso, o próximo paciente recebeu uma concentração de bupivacaína 0,0125\% menor.

b) Ineficaz: quando o escore analógico visual de dor foi maior que $10 \mathrm{~mm}$. Neste caso, foram administrados $15 \mathrm{ml}$ de lidocaína a $2 \%$ e, após 30 minutos, foi realizada nova avaliação.

Caso os escores analógicos visuais tenham sido iguais ou menores que $10 \mathrm{~mm}$, a concentração da bupivacaína foi considerada ineficaz e o próximo paciente recebeu uma concentração 0,0125\% maior. Caso o escore analógico visual de dor tenha persistido acima de $10 \mathrm{~mm}$, o caso foi rejeitado e a mesma concentração de bupivacaína foi utilizada no paciente seguinte.

As concentrações eficazes e ineficazes foram comparadas pelo teste $t$ de Student não pareado. Os escores analógicos visuais de dor foram comparados, entre as regiões operadas, pelo teste de Kruskal-Wallis. O nível de significância foi de $5 \%$.

\section{Estudo /I}

Foram estudados 23 pacientes de ambos os sexos, com idades entre 20 e 58 anos, submetidos a cirurgias ortopédicas Vol. 51, N 5, Setembro - Outubro, 2001 
eletivas sobre a perna, tornozelo ou pé. Os pacientes foram posicionados em decúbito lateral, sobre o membro a ser operado e receberam 12 a $15 \mathrm{mg}$ de bupivacaína hiperbárica a $0,5 \%$ no espaço subaracnóideo, através de uma agulha tipo Quincke calibre 27G, introduzida por acesso mediano, no espaço $L_{4}-L_{5}$. Uma agulha de Touhy calibre $16 \mathrm{G}$ foi introduzida no mesmo local e o espaço peridural foi localizado por perda de resistência à solução fisiológica. Um cateter $18 \mathrm{G}$ foi avançado 3 a $5 \mathrm{~cm}$ em direção cefálica, no espaço peridural. Não foi administrada dose-teste de anestésico local e a permeabilidade do cateter foi confirmada pela injeção de $1 \mathrm{ml}$ de solução fisiológica. O cateter foi protegido por um curativo oclusivo. A cirurgia teve início e o nível superior do bloqueio sensitivo foi testado com o toque da ponta de uma agulha calibre 22G, 30 minutos após a injeção subaracnóidea e a intervalos de 30 minutos, a partir de então. Os pacientes foram sedados durante o procedimento cirúrgico com doses repetidas de 2 mg de midazolam.

Após a regressão de 4 dermátomos do nível superior do bloqueio sensitivo (H0), $20 \mathrm{ml}$ de bupivacaína na concentração prevista pela resposta do paciente anterior, foram injetados através do cateter peridural, à velocidade de $4 \mathrm{ml}$. $\mathrm{min}^{-1}$ e infusão de bupivacaína na mesma concentração foi iniciada através de bomba volumétrica, à velocidade de 0,15 $\mathrm{ml} . \mathrm{kg}^{-1} \cdot \mathrm{h}^{-1}$. O primeiro paciente do estudo recebeu bupivacaína a $0,3 \%$. O intervalo entre as concentrações testadas foi de $0,1 \%$.

Um investigador que desconhecia a concentração da bupivacaína examinou os pacientes $4(\mathrm{H} 4), 8(\mathrm{H} 8)$ e $12(\mathrm{H} 12)$ horas após o início da infusão peridural e os seguintes dados foram coletados:

a) Dor em repouso e durante a mobilização do tornozelo e dos pododátilos, medida através de escala analógica visual de dor de $100 \mathrm{~mm}$ ( 0 = sem dor, 100 = pior dor imaginável);

b) Escores de Bromage durante mobilização ativa do membro operado: 0 = nenhum movimento do membro, 1 = flexão do tornozelo, 2 = flexão do tornozelo e joelho e 3 = flexão do tornozelo, joelho e quadril.

A eficácia analgésica de cada concentração de bupivacaína foi avaliada como segue:

a) Eficaz: quando, em todos os exames, o escore analógico visual foi menor ou igual a $10 \mathrm{~mm}$, em repouso e durante mobilização. Neste caso, o paciente seguinte recebeu uma concentração de bupivacaína $0,1 \%$ menor.

b) Ineficaz: quando, em um dos exames, o escore analógico visual em repouso ou durante mobilização foi maior que $10 \mathrm{~mm}$. Neste caso, foram administrados $15 \mathrm{ml}$ de bupivacaína a 0,5\% e, 30 minutos após, foi realizada nova avaliação.

Caso os escores analógicos visuais de dor em repouso ou durante a mobilização tenham sido iguais ou menores que 10 $\mathrm{mm}$, a concentração de estudo foi considerada ineficaz e o próximo paciente recebeu uma concentração $0,1 \%$ maior. Caso os escores analógicos visuais de dor tenham persistido acima de $10 \mathrm{~mm}$, o paciente foi rejeitado e a mesma concentração de bupivacaína foi utilizada no paciente seguinte. Aconcentração analgésica mínima da bupivacaína foi calculada pela fórmula de Massey e Dixon ${ }^{8}$.

O nível superior do bloqueio sensitivo e os escores de Bromage foram comparados entre as concentrações eficazes e ineficazes pelo teste de Mann-Whitney. O nível de significância foi de $5 \%$.

\section{RESULTADOS}

A tabela I mostra os dados demográficos de ambas as amostras.

Tabela I - Dados Demográficos das Amostras dos Estudos I e II

\begin{tabular}{lcc}
\hline Parâmetro & Estudo I & Estudo II \\
\hline Idade (anos) ${ }^{*}$ & $33,68 \pm 9,02$ & $36,25 \pm 10,81$ \\
Peso $(\mathrm{kg})^{*}$ & $73,63 \pm 11,40$ & $73,05 \pm 13,50$ \\
Altura $(\mathrm{cm})^{*}$ & $170,72 \pm 8,23$ & $168,9 \pm 11,01$ \\
Sexo ${ }^{* *}$ & & \\
$\quad$ Masculino & 23 & 13 \\
$\quad$ Feminino & 5 & 7 \\
\hline
\end{tabular}

* Média \pm DP

** Número de pacientes por categoria

\section{Estudo I}

A figura 1 mostra as respostas às diversas concentrações de bupivacaína testadas. Não foi possível calcular a CAM-AL da bupivacaína, uma vez que os dados não preencheram requisitos essenciais para aplicação da fórmula de Massey e Dixon. Houve 6 respostas eficazes, 15 respostas ineficazes e 7

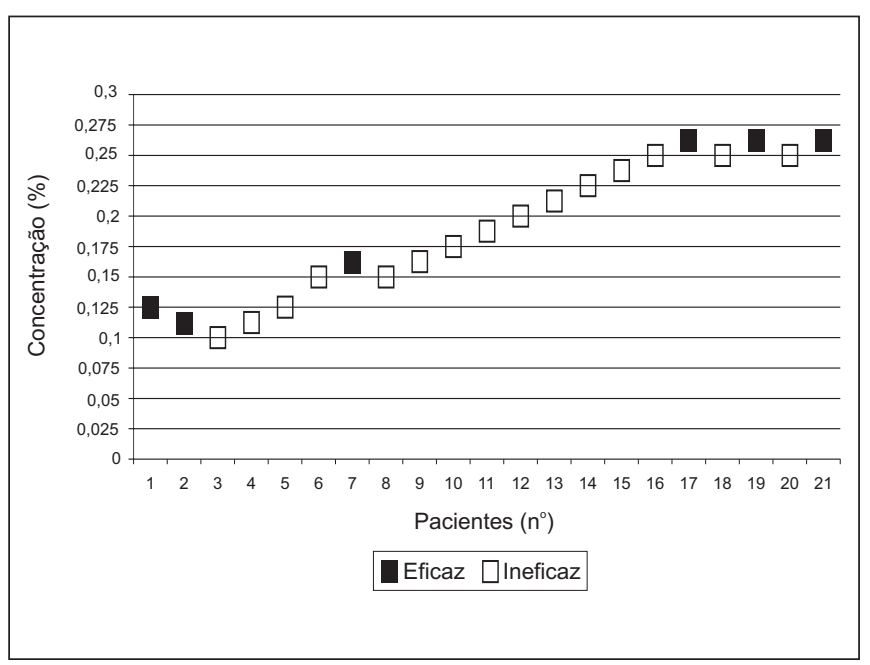

Figura 1 - Respostas às Concentrações de Bupivacaína Utilizadas no Estudo I 
rejeições. A concentração média de bupivacaína utilizada nesta amostra foi de $0,18 \pm 0,05 \%$. A concentração média eficaz de bupivacaína foi $0,19 \pm 0,07 \%$ e a ineficaz, 0,18 $\pm 0,055$ $\%(p>0,05)$. A mediana (extremos) dos escores analógicos visuais de dor antes da administração da bupivacaína foi 70 (25 e $100 \mathrm{~mm}$ ) e não diferiram significativamente segundo a região operada.

Estudo II

Afigura 2 representa as respostas às concentrações de bupivacaína testadas. Houve 11 respostas eficazes, 9 ineficazes e 3 rejeições. A concentração de bupivacaína foi considerada ineficaz em 3 pacientes em $\mathrm{H} 4,4$ pacientes em $\mathrm{H} 8$ e 2 pacientes em H12. ACAM-AL $\left(\mathrm{CE}_{50}\right)$ da bupivacaína foi estimada em $0,16 \%$, com intervalo de $95 \%$ de confiança entre $0,11 \%$ e $0,21 \%$. ACE ${ }_{95}$ foi estimada em $0,26 \%$, com limites de $95 \%$ de confiança em $0,21 \%$ e $0,31 \%$. Não houve diferença quanto ao nível superior do bloqueio sensitivo entre os pacientes que receberam concentrações eficazes e ineficazes da bupivacaína. As concentrações eficazes produziram escores de Bromage significativamente mais baixos do que as ineficazes (Tabelas II e III).

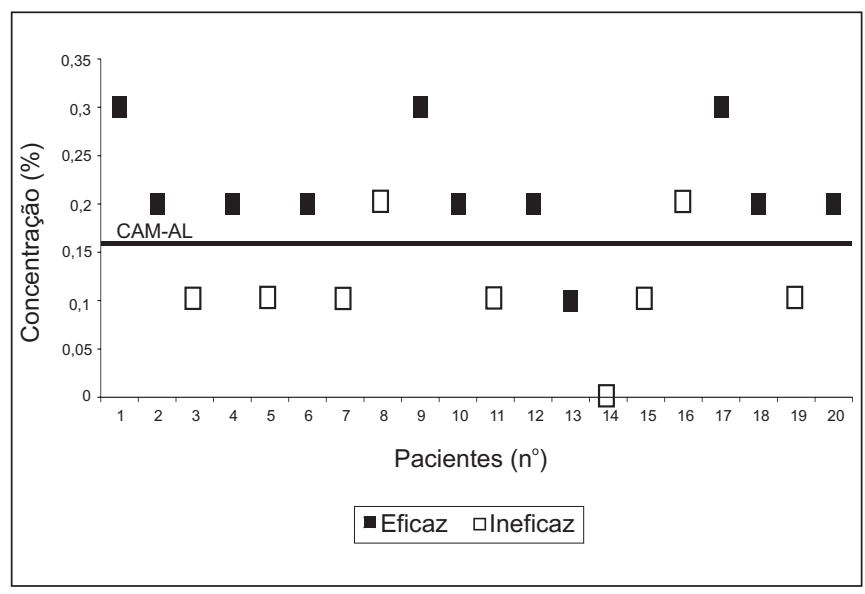

Figura 2 - Concentração Analgésica Mínima (CAM-AL) da Bupivacaína
Tabela III - Percentuais de Pacientes Segundo o Escore de Bromage em Cada Momento do Período de Observação

\begin{tabular}{lllll}
\hline & 0 & 1 & 2 & 3 \\
\hline $\mathrm{H} 4$ & 55 & 15 & 10 & 20 \\
$\mathrm{H} 8$ & 45 & 15 & 10 & 15 \\
$\mathrm{H} 12$ & 35 & 15 & 0 & 15 \\
\hline
\end{tabular}

\section{DISCUSSÃO}

Apesar de não ter permitido o cálculo da CAM-AL da bupivacaína em cirurgias ortopédicas do membro inferior, o estudo I permitiu a estimativa das médias e dos desvios padrão necessários para o cálculo do tamanho da amostra para o estudo II. Como o desvio padrão de toda a amostra foi de $0,05 \%$ e houve um intervalo de $0,1 \%$ entre as concentrações eficazes entre o caso 8 e o caso 16, decidiu-se adotar o intervalo de $0,1 \%$ no estudo II. Como não existe método para o cálculo a priori do tamanho da amostra para o método de alocação seqüencial não aleatório, a estimativa é feita baseada no teste $t$ de Student para amostras independentes ${ }^{7}$. Estabeleceu-se que uma diferença significativa entre as concentrações eficazes e ineficazes deveria ser maior que o desvio padrão e menor que o intervalo de teste, ou seja, entre 0,06 e 0,09\%. Com estes dados e considerando $\alpha=0,05$ e $\beta=0,2$, estimou-se o tamanho da amostra entre 10 e 18 pacientes.

O estudo I permitiu também estimar a intensidade da dor pós operatória em cirurgias ortopédicas sobre o membro inferior. Uma vez que a mediana dos escores analógicos visuais de dor foi de $70 \mathrm{~mm}$, refletindo dor de moderada a forte intensidade, concluiu-se que o modelo utilizado naquele estudo, por expor os pacientes a dor operatória de tal intensidade, deveria ser abandonado. Por esta razão, optou-se por estudar a CAM-AL da bupivacaína utilizando a infusão contínua pós-operatória, após anestesia subaracnóidea e, uma vez que a CAM-AL varia de acordo com a intensidade do estímulo doloroso ${ }^{11,12}$, restringiu-se a amostra do estudo II a cirurgias realizadas sobre a perna, tornozelo e pé.

Embora a concentração eficaz em $95 \%$ dos pacientes $\left(\mathrm{CE}_{95}\right)$ seja mais relevante para a prática clínica, a concentração eficaz em $50 \%$ dos pacientes $\left(\mathrm{CE}_{50}\right)$ é uma ferramenta mais útil de pesquisa, dada a sua posição na curva de concentra-

Tabela II - Nível Superior do Bloqueio Sensitivo e Escores de Bromage nos Diversos Momentos do Período de Observação: Comparação entre as Concentrações Eficazes e Ineficazes de Bupivacaína

\begin{tabular}{|c|c|c|c|c|}
\hline & \multicolumn{2}{|c|}{ Nível superior do bloqueio sensitivo } & \multicolumn{2}{|c|}{ Escores de Bromage } \\
\hline & Eficaz & Ineficaz & Eficaz & Ineficaz \\
\hline Após bloqueio subaracnóideo & $\mathrm{T} 4[\mathrm{~T} 1 ; \mathrm{T} 7]$ & $\mathrm{T} 2[\mathrm{~T} 1 ; \mathrm{T} 6]$ & $0[0 ; 0]$ & $0[0 ; 0]$ \\
\hline $\mathrm{HO}$ & $\mathrm{T} 8[\mathrm{~T} 4 ; \mathrm{T} 11]$ & T6 $[\mathrm{T} 5 ; \mathrm{T} 10]$ & $0[0 ; 0]$ & $0[0 ; 0]$ \\
\hline $\mathrm{H} 4$ & T12 [T6; L2] & T12 [T10; L3] & $2[0 ; 3]$ * & $0[0 ; 2]$ \\
\hline $\mathrm{H} 8$ & T12 [T6; L2] & T11,5 [T9; L2] & $0[0 ; 0]$ * & $0[0 ; 3]$ \\
\hline $\mathrm{H} 12$ & T12 [T6; L2] & L2 [T12; L4] & $0[0 ; 0]$ * & $0[0 ; 3]$ \\
\hline
\end{tabular}

${ }^{*} p<0,05$ comparado às concentrações eficazes 
ção-resposta dos anestésicos locais. $\mathrm{ACE}_{50}$ corresponde ao ponto onde a inclinação é máxima, de tal forma que intervenções que alteram a posição da curva de concentração-resposta são facilmente detectadas pela alteração dos valores da $C E_{50}$. Por outro lado, a $C E_{95}$ localiza-se na porção achatada superior da curva de concentração-resposta, de tal forma que somente grandes alterações da posição desta curva serão perceptíveis pela análise da $C E_{95}$.

ACAM-AL foi calculada pelo método de alocação seqüencial não aleatória descrito por Massey e Dixon ${ }^{8}$. Segundo o método, o intervalo entre as concentrações testadas deve ser menor que dois desvios padrão da média(DP). No estudo II, o intervalo entre as concentrações testadas foi de $0,1 \%$, ou seja 1,66 DP. A robustez do teste t foi estimada em 0,92, de tal forma que, com a amostra final de 20 pacientes, a possibilidade de erro tipo II, foi de 0,08.

Entre os pacientes que receberam concentrações de bupivacaína consideradas ineficazes, o nível superior do bloqueio sensitivo, medido pelo toque da ponta de uma agulha esteve entre $T_{10}$ e $L_{4}$, demonstrando que a ausência de sensibilidade medida por este método não impede que os pacientes refiram dor no território inervado por $\mathrm{L}_{5}$ e $\mathrm{S}_{1}$. Isto está de acordo com resultados de outros estudos, em que somente concentrações elevadas de anestésicos locais produzem bloqueio sensitivo completo das raízes de $L_{5}$ e $S_{1}{ }^{13-16}$. A razão para isto é que as raízes de $\mathrm{L}_{5}$ e $\mathrm{S}_{1}$ são mais calibrosas, o que prolonga a latência do bloqueio sensitivo e motor e diminui sua intensidade ${ }^{17}$. Em estudo anterior, a bupivacaína a $0,5 \%$, associada ou não ao fentanil por via peridural ou venosa, não proporcionou anestesia completa e imobilidade no território inervado pelas raízes $L_{4}, L_{5}$ e $S_{1}{ }^{18}$.

Nos estudos em analgesia do primeiro estágio do trabalho de parto, a CAM-AL da bupivacaína situou-se entre 0,048 e $0.14 \%^{1-7,10-12}$. No presente estudo, a CAM-AL da bupivacaína foi estimada em $0,16 \%$. Razões para esta diferença podem residir no tipo de dor (somática, neste estudo e visceral nos demais), na intensidade da dor gerada por cirurgias ortopédicas da perna, tornozelo e pé e no maior calibre das raízes $L_{5}$ e $S_{1}$, em comparação com as raízes de $T_{10}$ a $L_{1}$, envolvidas na dor do primeiro estágio do trabalho de parto.

Percentuais elevados de pacientes apresentaram bloqueio motor intenso durante o período de observação, sendo maior entre os pacientes que tiveram analgesia eficaz. Isto pode ser resultado do efeito cumulativo da infusão de bupivacaína. Em outros estudos, infusão prolongada de bupivacaína a $0,125 \%$ à velocidade de $0,15 \mathrm{ml} \cdot \mathrm{kg}^{-1} \cdot \mathrm{h}^{-1}$ não se associaram com prevalência de bloqueio motor semelhante à deste estudo ${ }^{19-21}$. Embora a bupivacaína peridural tenha sido administrada durante o período de recuperação do bloqueio subaracnóideo, é possível que tenha retardado o eliminação da bupivacaína do espaço subaracnóideo, já que esta depende de difusão extradural do anestésico local e é guiada por gradiente de concentração, ao mesmo tempo em que tenha ocorrido passagem subaracnóidea da bupivacaína administrada no espaço peridural, intensificando o bloqueio subaracnóideo ${ }^{22}$, mesmo não tendo aumentado o nível superior do bloqueio sensitivo após a administração peridural da bupivacaína.

Assim, embora a infusão contínua após bloqueio subaracnóideo tenha permitido o cálculo da CAM-AL da bupivacaína em cirurgias ortopédicas sobre perna, tornozelo e pé, é possível que este modelo não seja útil para investigar os efeitos sobre o bloqueio motor da concentração analgésica local mínima da bupivacaína.

\section{Minimum Analgesic Concentration of Bupivacaine After Continuous Epidural Infusion Following Spinal Anesthesia in the Postoperative Period of Leg, Ankle and Foot Surgery}

Getúlio Rodrigues de Oliveira Filho, M.D., Nilton Gesser, M.D., Márcia Regina Ghellar, M.D., Ranulfo Goldschmidt, M.D., Adilson José Dal Mago, M.D.

\section{INTRODUCTION}

Minimal analgesic concentration of a local anesthetic (MAC-LA) is the median effective concentration of a local anesthetic during the first stage of labor and corresponds to the concentration infused in the epidural space that will induce analgesia in $50 \%$ of patients $\left(E_{50}\right)^{1}$. The obstetric MAC-LA calculation model has allowed the establishment of the relative potency of different local anesthetics ${ }^{1-3}$ and the effect of simultaneous administration of epidural opioids ${ }^{4-7}$.

MAC-LA is calculated by the non randomized sequential allocation method described by Massey and Dixon and local anesthetic concentration administered to each patient is a function of previous patient's response ${ }^{1,8,9-11}$. Some advantages of this method are a $30 \%$ to $70 \%$ decrease in sample size as compared to multi-group studies with fixed local anesthetic concentrations, in addition to reducing the number of patients submitted to inadequate analgesia ${ }^{1,8}$.

Lower limb orthopedic surgeries cause moderate to severe postoperative pain, thus justifying continuous or intermittent epidural infusion of local anesthetics associated or not to opioids in the immediate postoperative period. However, the minimum analgesic concentration of local anesthetics for this type of surgery has not yet been defined. Knowing the MAC-LA of such anesthetic agents in lower limb orthopedic surgeries may be useful to compare relative potencies and to study the effects of adjuvant drugs.

This study aimed at determining bupivacaine's MAC-LA for continuous epidural infusion in the postoperative period of lower limb orthopedic surgeries.

A pilot study (Study I) was carried out to determine sample size, postoperative pain visual analog scores for lower limb surgeries and the intervals between bupivacaine concentrations to be used for MAC-LA determination (Study II). 


\section{METHODS}

Both studies were approved by Hospital Governador Celso Ramos Medical Ethics Committee and the informed consents of all patients were obtained.

All patients were classified as physical status ASAI. Patients aged below 18 or above 65 years, non-cooperative, with neurological or psychiatric diseases, drug addicted, with liver or kidney diseases, diabetes mellitus and plaster immobilization of the operated extremity were excluded from the study.

Both studies were prospective and double-blind.

Patients were premedicated with oral diazepam ( 0.1 to 0.15 $\left.\mathrm{mg} . \mathrm{kg}^{-1}\right) 60$ to 90 minutes before surgery. Peripheral venoclysis was installed for saline infusion at a rate of $2 \mathrm{ml} . \mathrm{kg}^{-1} \cdot \mathrm{h}^{-1}$. Monitoring consisted of cardioscope, non invasive blood pressure and pulse oximetry.

\section{Study $I$}

Participated in this study 28 patients aged 19 to 56 years, of both genders submitted to lower limb orthopedic surgeries (hip and femur: 4 patients; knee: 11 patients; leg, ankle and foot: 13 patients). An epidural catheter was placed at $L_{4}-L_{5}$ and advanced 3 to $5 \mathrm{~cm}$ in the cephalad direction. Anesthesia was induced with propofol ( 2 to $2.5 \mathrm{mg} \cdot \mathrm{kg}^{-1}$ ) and maintained with $50 \%$ nitrous oxide and isoflurane under manually controlled ventilation through a laryngeal mask. At recovery, visual analog scores were recorded on a $100 \mathrm{~mm}$ scale $(0=$ no pain, $100=$ the worst imaginable pain), according to previous instruction and training. If the visual analog score was above $10 \mathrm{~mm}$, patients would receive $20 \mathrm{ml}$ bupivacaine in the adequate concentration through the epidural catheter. The first patient received $0.125 \%$ bupivacaine and the following received $0.0125 \%$ more or less, according to previous patient's response, as described below.

Bupivacaine's analgesic concentration efficacy was evaluated 30 minutes after administration by an observer unaware of the concentration in use, and was classified as:

a) Effective - when pain visual analog score was lower than or equal to $10 \mathrm{~mm}$. In this case, the next patient received bupivacaine in a concentration reduced in $0.0125 \%$;

b) Ineffective - when pain visual analog score was higher than $10 \mathrm{~mm}$. In this case, $15 \mathrm{ml}$ of $2 \%$ lidocaine were administered and a new evaluation was performed $30 \mathrm{mi}-$ nutes later.

Case visual analog scores were equal to or lower than $10 \mathrm{~mm}$, bupivacaine's concentration was considered ineffective and the next patient would received $0.0125 \%$ more. If the visual analog score remained above $10 \mathrm{~mm}$, the case was rejected and the next patient would receive the same bupivacaine's concentration.

Effective and ineffective concentrations were compared by non paired Student's $t$ test. Pain visual analog scores in operated sites were compared by Kruskal-Wallis test. Significance level was established to $5 \%$.

\section{Study II}

Participated in this study 23 patients of both genders, aged 20 to 58 years, submitted to elective leg, ankle or foot orthopedic surgery. Patients were placed in the lateral position over the operated limb and received 12 to $15 \mathrm{mg}$ of $0.5 \%$ hyperbaric bupivacaine in the subarachnoid space through a $27 \mathrm{G}$ Quincke needle introduced by median access at $L_{4}-L_{5}$ level. A $16 G$ Tuohy needle was introduced in the same place and the epidural space was located by the loss of resistance to saline test. An $18 \mathrm{G}$ epidural catheter was then introduced 3 to $5 \mathrm{~cm}$ in the cephalad direction. No local anesthetics test dose was administered and catheter patency was confirmed by $1 \mathrm{ml} \mathrm{sa-}$ line injection. An occlusive dressing protected the catheter. Surgery was started and upper sensory block level was tested by pinprick ( $22 \mathrm{G}$ needle) 30 minutes after spinal injection and at 30-minute intervals thereof. Patients were sedated during surgery with repeated doses of $2 \mathrm{mg}$ midazolam.

After a 4-dermatome regression of upper sensory block (HO), $20 \mathrm{ml}$ bupivacaine in the concentration determined by the previous patient's response were injected through the epidural catheter at a rate of $4 \mathrm{ml} \cdot \mathrm{min}^{-1}$, followed by bupivacaine infusion in the same concentration through a volumetric pump at a rate of $0.15 \mathrm{ml} \cdot \mathrm{kg}^{-1} \cdot \mathrm{h}^{-1}$. The first patient received $0.3 \%$ bupivacaine. The intervals between tested concentrations were $0.1 \%$.

An investigator unaware of the bupivacaine's concentration used evaluated the patients $4(\mathrm{H} 4), 8(\mathrm{H} 8)$ and $12(\mathrm{H} 12)$ hours after epidural infusion and the following data were collected:

a) Pain at rest and during ankle and toes movement, measured through a $100 \mathrm{~mm}$ pain visual analog score $(0=$ no pain; 100 = the worst imaginable pain);

b) Bromage scores for active movement of the operated limb: 0 = no movement, 1 = ankle flexion, 2 = ankle and knee flexion, and $3=$ ankle, knee and hip flexion.

Bupivacaine analgesic efficacy was evaluated as follows:

a) Effective when, in all evaluations, visual analog scores were lower than or equal to $10 \mathrm{~mm}$ at rest and during movement. In this case, the next patient would receive a bupivacaine's concentration $0.1 \%$ lower;

b) Ineffective when, at least in one evaluation, visual analog score at rest or during movement was higher than 10 $\mathrm{mm}$. In this case, $15 \mathrm{ml}$ of $0.5 \%$ bupivacaine were administered and a new evaluation was performed after 30 minutes. If pain visual analog score at rest or during movement was equal to or lower than $10 \mathrm{~mm}$, the concentration studied was considered ineffective and the next patient would receive $0.1 \%$ more bupivacaine. 
Case pain visual analog score persisted above $10 \mathrm{~mm}$, patient was rejected and the same bupivacaine's concentration was administered to the next patient.

Bupivacaine's minimum analgesic concentration was calculated by Massey and Dixon's formula ${ }^{8}$.

Upper sensory block level and Bromage scores were compared between effective and ineffective concentrations by Mann-Whitney test. Significance level was established to $5 \%$.

\section{RESULTS}

Demographics data of both samples are shown in table I.

Table I - Demographics of Studies I and II Samples

\begin{tabular}{lcc}
\hline Parameter & Study I & Study II \\
\hline Age (years) * & $33.68 \pm 9.02$ & $36.25 \pm 10.81$ \\
Weight $(\mathrm{kg}){ }^{*}$ & $73.63 \pm 11.40$ & $73.05 \pm 13.50$ \\
Height $(\mathrm{cm}){ }^{*}$ & $170.72 \pm 8.23$ & $168.9 \pm 11.01$ \\
Gender ** & & \\
Male & 23 & 13 \\
Female & 5 & 7 \\
\hline
\end{tabular}

* Mean $\pm \mathrm{SD}$

${ }^{* *}$ Number of patients by category

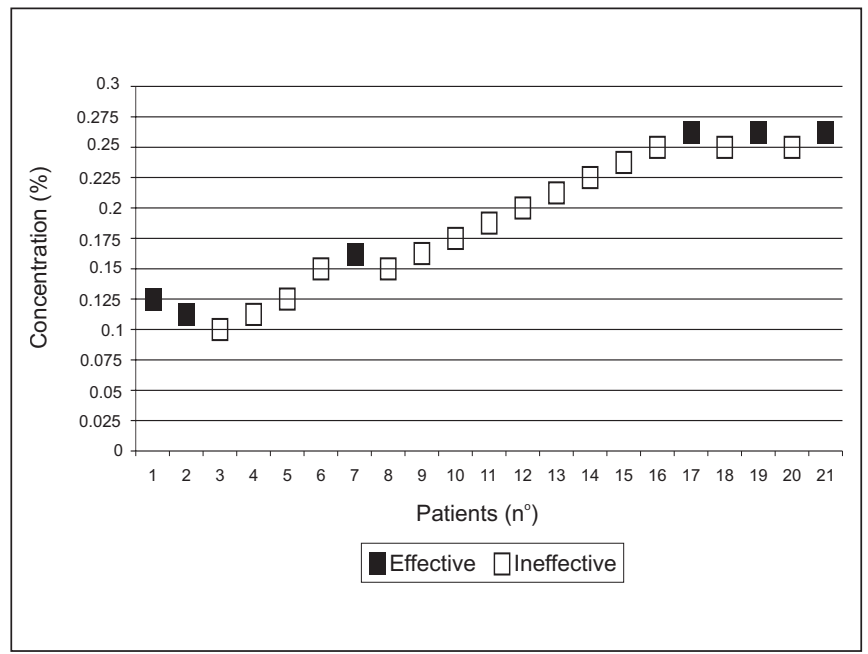

Figure 1 - Following Spinal Anesthesia in the Postoperative Period of Leg, Ankle and Foot Surgery

\section{Study 1}

Figure 1 shows the responses to different bupivacaine concentrations. Bupivacaine's MAC-LA could not be calculated because data did not meet essential Massey and Dixon formula's requirements. There were 6 effective responses, 15 ineffective and 7 rejections. Mean bupivacaine concentration in this sample was $0.18 \% \pm 0.05 \%$. Mean effective bupivacaine concentration was $0.19 \% \pm 0.07 \%$ and mean ineffective concentration was $0.18 \% \pm 0.055 \%(p>0.05)$. Median pain visual analog score (extremes) before bupivacaine administration was 70 ( 25 and $100 \mathrm{~mm}$ ) and did not significantly differ according to the operated site.

\section{Study II}

Figure 2 shows the responses to the tested bupivacaine's concentrations. There were 11 effective responses, 9 ineffective and 3 rejections. Bupivacaine's concentrations were considered ineffective in 3 patients in $\mathrm{H} 4,4$ patients in $\mathrm{H} 8$ and 2 patients in $\mathrm{H} 12$. Bupivacaine's MAC-LA( $\left(\mathrm{EC}_{50}\right)$ was calculated in $0.16 \%$, with a $95 \%$ confidence interval between $0.11 \%$ and $0.21 \%$. EC $E_{95}$ was calculated in $0.26 \%$, with $95 \%$ confidence intervals between $0.21 \%$ and $0.31 \%$. There were no upper sensory block level differences between patients receiving bupivacaine effective and ineffective concentrations.

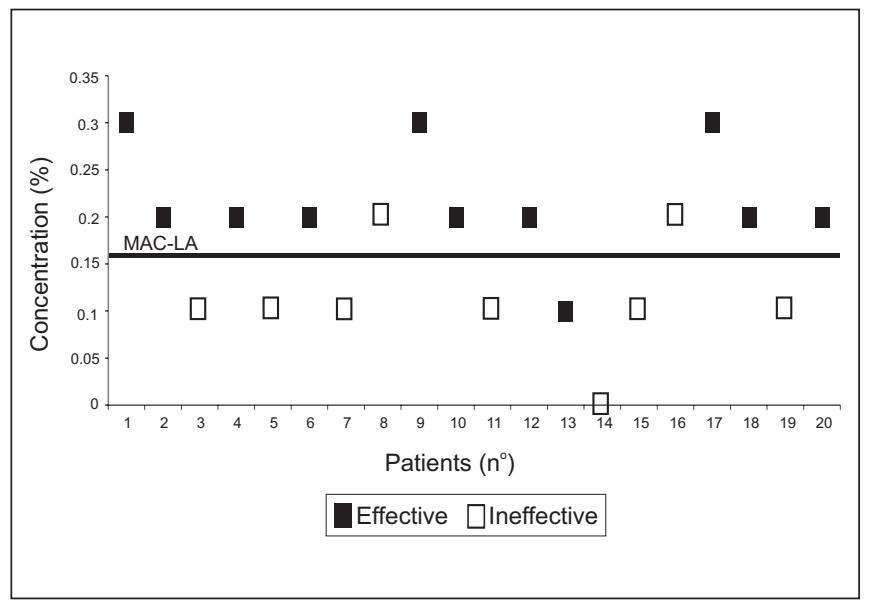

Figure 2 - Minimum Analgesic Concentration of Bupivacaine Concentration for Continuous Epidural Infusion

Table II - Upper Sensory Block Level and Bromage Scores in Different Observation Period Moments: Comparison Between Effective and Ineffective Bupivacaine Concentrations

\begin{tabular}{lcccc}
\hline & \multicolumn{2}{c}{ Upper sensory block level } & \multicolumn{2}{c}{ Bromage Scores } \\
\hline After spinal block & Effective & Ineffective & Effective & Ineffective \\
H0 & T4 [T1; T7] & T2 [T1; T6] & $0[0 ; 0]$ & $0[0 ; 0]$ \\
H4 & T8 [T4; T11] & T6 [T5; T10] & $0[0 ; 0]$ & $0[0 ; 0]$ \\
H8 & T12 [T6; L2] & T12 [T10; L3] & $2[0 ; 3]^{*}$ & $0[0 ; 2]$ \\
H12 & T12 [T6; L2] & T11,5 [T9; L2] & $0[0 ; 0]^{*}$ & $0[0 ; 3]$ \\
\hline
\end{tabular}

${ }^{*} p<0.05$ as compared to effective concentrations 
Effective concentrations produced significantly lower Bromage scores as compared to ineffective concentrations ( Tables II and III).

Table III - Patients Percentages According to Bromage Score in Each Observation Period Moment

\begin{tabular}{lcccc}
\hline & 0 & 1 & 2 & 3 \\
\hline $\mathrm{H} 4$ & 55 & 15 & 10 & 20 \\
$\mathrm{H} 8$ & 45 & 15 & 10 & 15 \\
$\mathrm{H} 12$ & 35 & 15 & 0 & 15 \\
\hline
\end{tabular}

\section{DISCUSSION}

Although not allowing bupivacaine's MAC-LA calculation in lower limb orthopedic surgeries, study I provided mean and standard deviation estimates needed to calculate study II sample size. Since standard deviation for the whole sample was $0.05 \%$ and there has been a $0.1 \%$ interval between effective concentrations of case 8 and case 16 , this $0.1 \%$ interval was adopted in study II. Since there is no method for a priori sample size calculation for the non randomized sequential allocation method, estimates were based on Student's $t$ test for independent samples ${ }^{7}$. It was established that a significant difference between effective and ineffective concentrations should be higher than the standard deviation and lower than the test interval, that is, between $0.06 \%$ and $0.09 \%$. With the above data and considering $\alpha=0.05$ and $\beta=0.2$, sample size was estimated between 10 and 18 patients.

Study I has also allowed postoperative pain intensity estimates in lower limb orthopedic surgeries. Since median pain visual analog score was $70 \mathrm{~mm}$, reflecting moderate or severe pain, the conclusion was that the model should be abandoned for exposing patients to such severe postoperative pain. For this reason, we decided to study bupivacaine's MAC-LA using postoperative continuous infusion after spinal anesthesia and, since MAC-LA varies according to the painful stimulus intensity ${ }^{11,12}$, study II sample was limited to leg, ankle and foot surgeries.

Although the effective concentration in $95 \%$ of patients $\left(E_{95}\right)$ be more relevant for clinical practice, the effective concentration in $50 \%$ of patients $\left(E_{50}\right)$ is a more useful research tool due to its position in local anesthetics concentration-response curve. $\mathrm{EC}_{50}$ corresponds to the point were there is a maximum slope so that any factor changing the concentration-response curve position is easily detected by changes in $\mathrm{EC}_{50}$ values. On the other hand, $\mathrm{EC}_{95}$ is located at the upper flat portion of the concentration-response curve in a way that only major changes in its position will be noticed by $\mathrm{EC}_{95}$ analysis.

MAC-LAwas calculated by the non randomized sequential allocation method described by Massey and Dixon ${ }^{8}$. According to this method, the interval between tested concentrations should be lower than two standard deviations (SD). In study II, the interval between tested concentrations was $0.1 \%$, or 1.66 SD. Robustness of test $t$ was estimated as 0.92 , so that with the final sample of 20 patients, the possibility of type II error was 0.08 .

Among patients receiving ineffective bupivacaine concentrations, upper sensory block level measured by pinprick remained between $T_{10}$ and $L_{4}$, showing that the lack of sensitivity measured by such method does not prevent patients from referring pain in the territory innervated by $L_{5}$ and $S_{1}$. This is in line with other studies where only high local anesthetic concentrations produced a complete sensory block of $L_{5}$ and $S_{1}$ roots ${ }^{13-16}$. This is because $L_{5}$ and $S_{1}$ roots are thicker, thus prolonging sensory and motor block onset and decreasing their intensity ${ }^{17}$. In a previous study, $0.5 \%$ bupivacaine, associated or not to epidural or intravenous fentanyl, has not provided total anesthesia and immobility in the territory innervated by $L_{4}, L_{5}$ and $S_{1}$ roots ${ }^{18}$.

In studies of first stage labor analgesia, bupivacaine's MAC-LA was estimated between $0.048 \%$ and $0.14 \%{ }^{1-7,10-12}$. In our study, bupivacaine's MAC-LA was estimated in $0.16 \%$. Reasons for such difference may be the type of pain (somatic in this study and visceral in the others), pain intensity generated by leg, ankle and foot orthopedic surgeries and larger $L_{5}$ and $S_{1}$ roots as compared to $T_{10}$ to $L_{1}$ roots involved in first stage labor pain.

A large number of patients presented with intense motor block during the observation period, and this was even higher among patients with effective analgesia. This may be due to the cumulative effect of bupivacaine infusion. In other studies, prolonged $0.125 \%$ bupivacaine infusions at a rate of 0.15 $\mathrm{ml} . \mathrm{kg}^{-1} \cdot \mathrm{h}^{-1}$ were not associated to a prevalence of motor block similar to our study ${ }^{19-21}$. Although bupivacaine's administration took place during the spinal block recovery period, it might have delayed bupivacaine elimination from the spinal space because it depends on extradural local anesthetic spread and is forced by concentration gradient; at the same time there might have been spinal spread of epidural bupivacaine, thus intensifying spinal block ${ }^{22}$ even with no increase in upper sensory block level after epidural bupivacaine administration.

So, although continuous infusion after spinal block having allowed bupivacaine's MAC-LA calculation in leg, ankle and foot orthopedic surgeries, it is possible that this model will not be useful to investigate bupivacaine's minimum analgesic concentration effects on motor block.

\section{REFERÊNCIAS - REFERENCES}

01. Columb MO, Lyons G - Determination of the minimum local analgesic concentrations of epidural bupivacaine and lidocaine in labor. Anesth Analg, 1995;81:833-837.

02. Capogna G, Celleno D, Fusco P et al - Relative potencies of bupivacaine and ropivacaine for analgesia in labour. $\mathrm{Br} \mathrm{J}$ Anaesth, 1999;82:371-373.

03. Polley LS, Columb MO, Naughton NN et al - Relative analgesic potencies of ropivacaine and bupivacaine for epidural analgesia in labor: implications for therapeutic indexes. Anesthesiology, 1999;90:944-950. 
04. Columb MO, Polley LS, Wagner DS et al - Reduction in the minimum local analgesic concentration (MLAC) of bupivacaine by epidural sufentanil is dose dependent. European $\mathrm{J}$ Anaesthesiol, 1997;14:549-550.

05. Polley LS, Columb MO, Wagner DS et al - Dose-dependent reduction of the minimum local analgesic concentration of bupivacaine by sufentanil for epidural analgesia in labor. Anesthesiology, 1998;89:626-632.

06. Polley LS, Columb MO, Lyons $\mathrm{G}$ et al - The effect of epidural fentanyl on the minimum local analgesic concentration of epidural chloroprocaine in labor. Anesth Analg, 1996;83: 987-990.

07. Polley LS, Columb MO, Naughton NN et al - Effect of intravenous vs epidural fentanyl on the minimum local analgesic concentration (MLAC) of epidural bupivacaine in labor. Anesthesiology, 1999;90:25A.

08. Dixon WJ, Massey FJ - Introduction to Statistical Analysis. $4^{\text {th }}$ Ed, New York, McGraw-Hill, 1983:428-439.

09. Wulf HF - Up-down sequential allocation technique to investigate the influence of opioids on the efficacy of epidural local anesthetics in labor pain. Anesthesiology, 1999;90:1788.

10. Columb MO, Polley LS - Up-down sequential allocation technique to investigate the influence of opioids on the efficacy of epidural local anesthetics in labor pain. Anesthesiology, 1999;90:1788-1789.

11. Columb MO, Lyons G, Polley LS - Bupivacaine requirements for labor analgesia. Anesthesiology, 1999;90:73A.

12. Capogna G, Celleno D, Lyons G et al - Minimum local analgesic concentration of extradural bupivacaine increases with progression of labour. Br J Anaesth, 1998;80:11-13.

13. Galindo A, Benavides O, De Munos SO et al - Comparison of anesthetic solutions used in lumbar and caudal peridural anesthesia. Anesth Analg, 1978;57:175-179.

14. Arendt-Nielsen L, Oberg B, Bjerring P - Quantitative assessment of extradural bupivacaine analgesia. $\mathrm{Br} J$ Anaesth, 1990;65:633-638.

15. Axelsson K, NydahI PA, Philipson L et al - Motor and sensory blockade after epidural injection of mepivacaine, bupivacaine and etidocaine: a double-blind study. Anesth Analg, 1989;69:739-747.

16. Lund C, Hansen OB, Kehlet H - Effect of epidural $0.25 \%$ bupivacaine on somatosensory evoked potentials to dermatomal stimulation. Reg Anesth, 1989;14:72-77.

17. Galindo A, Hernandez J, Benavides O et al - Quality of spinal extradural anaesthesia: the influence of spinal nerve root diameter. $\mathrm{Br} J$ Anaesth, 1975;47:41-47.

18. Oliveira $F^{\circ}$ GR, Pederneiras SG, Ghellar MR et al - Qualidade da anestesia das raízes L4, L5 e S1 com bupivacaína 0,5\% epidural: efeitos da associação de fentanil por via epidural ou venosa. Rev Bras Anestesiol, 1996;46:CBA013.

19. Rygnestad T, Borchgrevink PC, Eide E - Postoperative epidural infusion of morphine and bupivacaine is safe on surgical wards. Organisation of the treatment, effects and side-effects in 2000 consecutive patients. Acta Anaesthesiol Scand, 1997;41: 868-876.

20. Scott DA, Beilby DS, McClymont C - Postoperative analgesia using epidural infusions of fentanyl with bupivacaine. A prospective analysis of 1,014 patients. Anesthesiology, 1995;83: 727-737

21. Bogod DG, Rosen M, Rees GA - Extradural infusion of $0.125 \%$ bupivacaine at $10 \mathrm{~m} \cdot \mathrm{h}^{-1}$ to women during labour. $\mathrm{Br} \mathrm{J}$ Anaesth, 1987;59:325-330.

22. Stienstra R, Dahan A, Alhadi BZ et al - Mechanism of action of an epidural top-up in combined spinal epidural anesthesia. Anesth Analg, 1996;83:382-386.

Revista Brasileira de Anestesiologia

Vol. 51, № 5, Setembro - Outubro, 2001

\section{RESUMEN}

Oliveira Filho GR, Gesser N, Ghellar MR, Goldschmidt R, Dal Mago AJ - Concentración Analgésica Mínima de la Bupivacaína Durante Infusión Peridural Continua después de Bloqueo Subaracnóideo en el Período Pós-Operatorio de Cirugías Ortopédicas de la Pierna, Tobillo y Pie

Justificativa y Objetivos - La concentración analgésica mínima de un anestésico local (CAM-AL) corresponde a la concentración efectiva en $50 \%$ de las pacientes durante la primera parte del trabajo de parto. Puede ser utilizada para determinar la potencia relativa de diferentes agentes y estimar el efecto de drogas analgésicas co-administradas en el espacio peridural. El objetivo de este estudio fue el de determinar la CAM-AL de la bupivacaína para analgesia peridural de cirugías ortopédicas.

Método - Fue aplicada la técnica de alocación secuencial no aleatoria doblemente encubierta a 23 adultos sometidos a cirugías ortopédicas sobre la pierna, tobillo o pie. La anestesia constó de bloqueo subaracnóideo lumbar con bupivacaína hiperbárica. Un catéter peridural colocado en $L_{4}-L_{5}$ fue avanzado 3 a $5 \mathrm{~cm}$ en dirección cefálica. En período pós-operatorio inmediato, fueron administrados $20 \mathrm{ml}$ de bupivacaína seguida de infusión de $0.15 \mathrm{ml} \cdot \mathrm{kg}^{-1} \cdot \mathrm{h}^{-1}$, en la concentración apropiada. Puntos analógicos visuales de dolor y de Bromage fueron registrados después 4, 8 y 12 horas. La concentración fue considerada eficaz cuando los puntos de dolor fueron inferiores a $10 \mathrm{~mm}$ en todas las evaluaciones. La concentración inicial fue de 0,3\% y disminuyó o aumentó 0,1\% caso la respuesta del paciente anterior haya sido ineficaz o eficaz, respectivamente. La CAM-AL fue calculada por la fórmula de Massey y Dixon.

Resultados - La CAM-AL de la bupivacaína (límites de 95\% de confianza) fue de $0,16 \%(0,11 \%$ y $0,21 \%)$. Bloqueo motor intenso fue observado en la mayoría de los pacientes.

Conclusiones - Para una tasa de infusión de $0.15 \mathrm{ml} . \mathrm{kg}^{-1} . \mathrm{h}^{-1}$ la CAM-AL de bupivacaína fue de $0,16 \%$. No obstante, el modelo utilizado puede no haber sido adecuado para la evaluación de los efectos motores de las concentraciones testadas. 